\title{
Vogt Koyanagi Harada syndrome in a 15-year-old girl, steroids side effects and recurrences
}

\author{
Gamze Yıldırım ${ }^{\oplus}$, Cemal Çavdarlı ${ }^{\oplus}$, Emine Yıldız Özdemir ${ }^{\oplus}$, Mehmet Numan Alp \\ Department of Ophthalmology, Niğde Ömer Halisdemir Education and Research Hospital, Niğde, Turkey.
}

\begin{abstract}
Background. Vogt-Koyanagi-Harada Syndrome is rare in childhood and is usually seen between the 2nd and 5th decades. We present a 15-year-old girl with findings of incomplete Vogt-Koyanagi-Harada Syndrome.

Case. In the first visit, anterior chamber inflammation, vitritis, serous retinal detachment and papillitis were observed in her both eyes. She also had neurological symptoms such as a headache. During the systemic treatment period, some of the side effects related to steroids emerged. Additionally, the symptoms and findings of the disease relapsed while the steroid dose was reduced.

Conclusion. Early diagnosis and selection of an individualized appropriate treatment provided good clinical and visual results without any serious complications in our case.
\end{abstract}

Key words: Vogt-Koyanagi-Harada syndrome, steroid side effects, panuveitis, immunosuppressive agents.

Vogt-Koyanagi-Harada Syndrome (VKHS) is an autoimmune disease characterized by idiopathic, chronic and diffuse granulomatous uveitis. The syndrome also includes neurological, auditory and dermatological symptoms. The most common ocular findings of VKHS are iridocyclitis, vitritis, optic disc edema, and exudative retinal detachments. ${ }^{1}$ Although VKHS is rare in childhood and usually emerges between 20 and 50 years; the disease can be a potential cause of blindness as a result of uveitis in children. Major sequelae that led to visual impairment include cataracts, glaucoma, retinal pigment epithelial changes, choroid neovascular membranes, and the scars of retinachoroiditis. ${ }^{1,2}$ The ocular complications are more severe in children than in adults. Thus, early and aggressive therapy with corticosteroids and / or other immunosuppressive agents have been involved in the treatment of children with VKHS. ${ }^{3}$ Clinical features of VKH disease

Gamze Yildirım

gmz_y_06@hotmail.com

Received 8th April 2020, revised 7th July 2020,

19th July 2020, 6th September 2020,

accepted 16th October 2020. have been identified well in adults, however, only case reports exist for children (Table I). We report a 15-year-old girl who presented with relapsed VKHS while the systemic steroid therapy was reduced for sparing from the side effects of steroids.

\section{Case Report}

A 15-year-old girl presented to our clinic with decreased vision in both eyes. In her anamnesis, she had a headache that had not responded to analgesics (paracetamol and ibuprofen, respectively) and continued for two days before one week of admission, and additionally, her vision decreased progressively two days before the admission. The patient underwent complete ophthalmologic examination including biomicroscopy, tonometry, and indirect ophthalmoscopy. Fundus fluorescein angiography (FFA) and ultrasonography were also performed. In the patient's examination, the best-corrected visual acuity (BCVA) was 20/100, bilaterally, according to the Snellen chart and intraocular pressure was $14 \mathrm{mmHg}$ in the right and $16 \mathrm{mmHg}$ in the left eye, respectively. 
Table I. Summary of the previous published reports of Vogt-Koyanagi-Harada syndrome (VKHS) in children in the last 10 years.

\begin{tabular}{|c|c|c|c|c|}
\hline Reference & Age & Sex & Clinical findings & Treatment \\
\hline \multirow{3}{*}{ AlQahtani et al. ${ }^{4}$} & \multirow{3}{*}{4} & \multirow{3}{*}{$\mathrm{M}$} & \multirow{3}{*}{$\begin{array}{l}\text { Band keratopathy, anterior uveitis, posterior synechiae, iris } \\
\text { atrophy, cataract, vitiligo and polisosis }\end{array}$} & $\begin{array}{l}\text { Corticosteroid } \\
\text { Cyclosporine }\end{array}$ \\
\hline & & & & Adalimumab \\
\hline & & & & Methotrexate \\
\hline Katsuyama et al. ${ }^{5}$ & 3 & $\mathrm{~F}$ & $\begin{array}{l}\text { Posterior synechiae, optic disk swelling, serous retinal } \\
\text { detachment }\end{array}$ & Corticosteroid \\
\hline Khan et al. ${ }^{6}$ & 16 & $\mathrm{~F}$ & Serous retinal detachment & Corticosteroid \\
\hline Takada et al. $^{7}$ & 3 & $\mathrm{M}$ & $\begin{array}{l}\text { Ciliary hyperemia, slight corneal oedema, keratic } \\
\text { precipitates, serous retinal detachment, aseptic meningitis }\end{array}$ & Corticosteroid \\
\hline Bilgiç et al. ${ }^{8}$ & 14 & M & Pale optic disc, retinal pigment atrophy, vitiligo & - \\
\hline Vergaro et al. ${ }^{9}$ & 12 & M & $\begin{array}{l}\text { Retinal vasculitis, retrocochlear hearing loss, aseptic } \\
\text { meningitis, cerebral ischemia }\end{array}$ & Corticosteroid \\
\hline Bušányová et al. ${ }^{10}$ & 11 & M & Granulomatous panuveitis, exsudative retinal detachment & Corticosteroid \\
\hline Hernandez et al. ${ }^{11}$ & 6 & M & $\begin{array}{l}\text { Cataract, panuveitis, optic nerve head edema, exudative } \\
\text { retinal detachment, vitiligo }\end{array}$ & Methotrexate \\
\hline \multirow{2}{*}{ Venaille et al. ${ }^{12}$} & \multirow{2}{*}{10} & \multirow{2}{*}{ M } & \multirow{2}{*}{$\begin{array}{l}\text { Anterior uveitis, optic disk swelling, serous retinal } \\
\text { detachment, aseptic meningitis alopecia, vitiligo }\end{array}$} & $\begin{array}{l}\text { Corticosteroid } \\
\text { Cyclosporine }\end{array}$ \\
\hline & & & & $\begin{array}{l}\text { Mycophenolate } \\
\text { mofetil }\end{array}$ \\
\hline Benfdil et al. ${ }^{13}$ & 14 & $\mathrm{~F}$ & $\begin{array}{l}\text { Uveitis, serous retinal detachment, choroidal } \\
\text { depigmentation, poliosis }\end{array}$ & Corticosteroid \\
\hline
\end{tabular}

Biomicroscopic examination revealed granulomatous anterior chamber reaction and vitritis in both eyes (Fig. 1). Additionally, the fundus examination revealed bilateral exudative retinal detachment in the posterior pole and hyperemic optic discs (Fig. 2). After the complementation of the clinical examination, multimodal-imaging was performed. Bilateral serous retinal detachment was also observed in the enhanced depth imaging of spectral

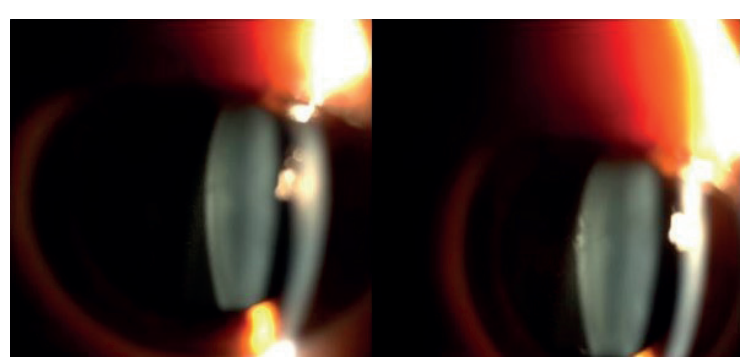

Fig. 1. Anterior segment images at the first visit: inflammatory cells in the anterior vitreous were observed in both eyes. domain optical coherence tomography (EDISD-OCT) scans (Fig. 3a). Subfoveal choroidal thickness of both eyes was measured in the first visit (401 $\mu, 412 \mu$ in the right eye and the left eye, respectively). FFA showed a starry sky appearance which is specific for VKHS (Fig. 4). Ocular B-scan ultrasonography showed serous retinal detachment and no scleritis (Fig. 2). Body temperature was $36.6^{\circ} \mathrm{C}$. Hemogram, sedimentation rate, C- Reactive Protein (CRP), and all of the routine biochemical values were within normal limits. The rheumatoid factor (RF), antinuclear antibody (ANA), anti-ds-DNA, Anti Smooth Muscle Antibody (ASMA) and Extractable Nuclear Antigen (ENA) profiles were negative. Treponema pallidum hemagglutination test and serous immunoglobulin $\mathrm{M}$ of varicella-zoster, herpes simplex and cytomegalovirus viruses were all negative. Lyme antibody test and brucella agglutination test were negative. Tuberculin skin test was negative, and her Enzyme-Linked 


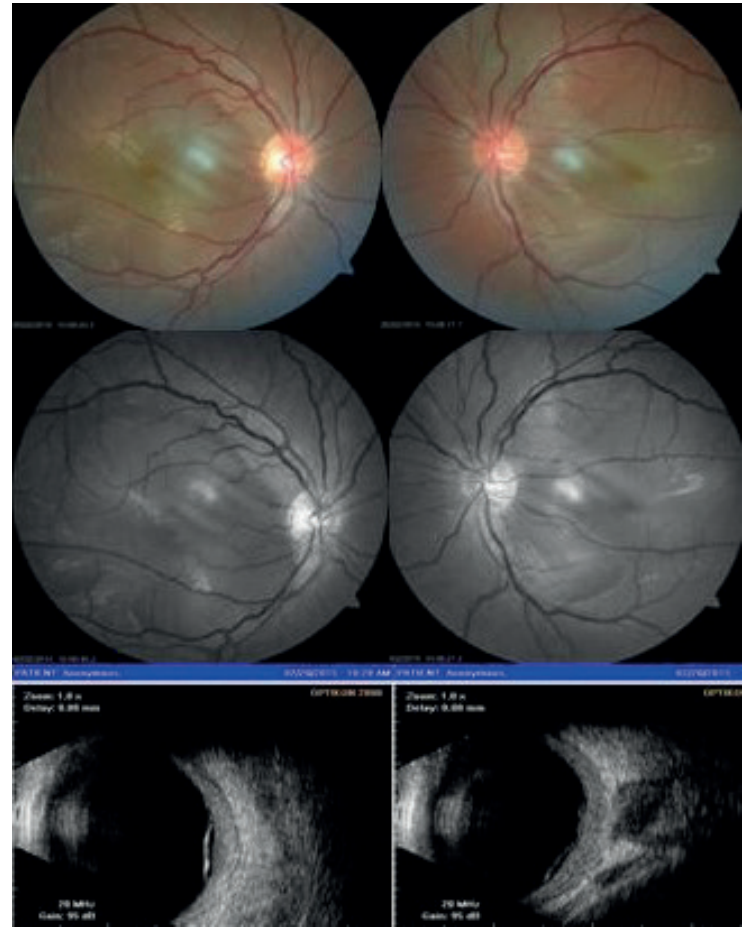

Fig. 2. Color photographs of the fundus and ocular B-scan ultrasonography images at the first visit.

Immunosorbent Assay (ELISA) of HIV was negative. Serum angiotensin-converting enzyme level was normal. Pathergy test was negative. Aseptic meningitis was reported as a result of neurology consultation. The patient had no tinnitus or hearing loss, and all audiology tests were normal. There was no pathological finding in the dermatology consultation. No pathology was observed on the chest X-ray.

The patient was hospitalized with a systemic and topical (for the inflammatory findings in the anterior chamber and vitreous) steroid treatment plan. After intravenous steroid treatment (methylprednisolone 1,000 $\mathrm{mg}$ for 3 days), oral steroid (prednisolone $1 \mathrm{mg} / \mathrm{kg}$ ) was continued. On the seventh day of the treatment, BCVA increased to 20/40 bilaterally, and her intraocular pressures were within normal limits (14 to $20 \mathrm{mmHg}$ ). Bilateral OCT images revealed decreased subretinal fluid (Fig. 3b). Bilateral BCVA of the patient was 20/20 at the control visit of the first month while she was under a $0.5 \mathrm{mg} / \mathrm{kg}$ oral prednisolone treatment. Furthermore, the SD-OCT image showed the
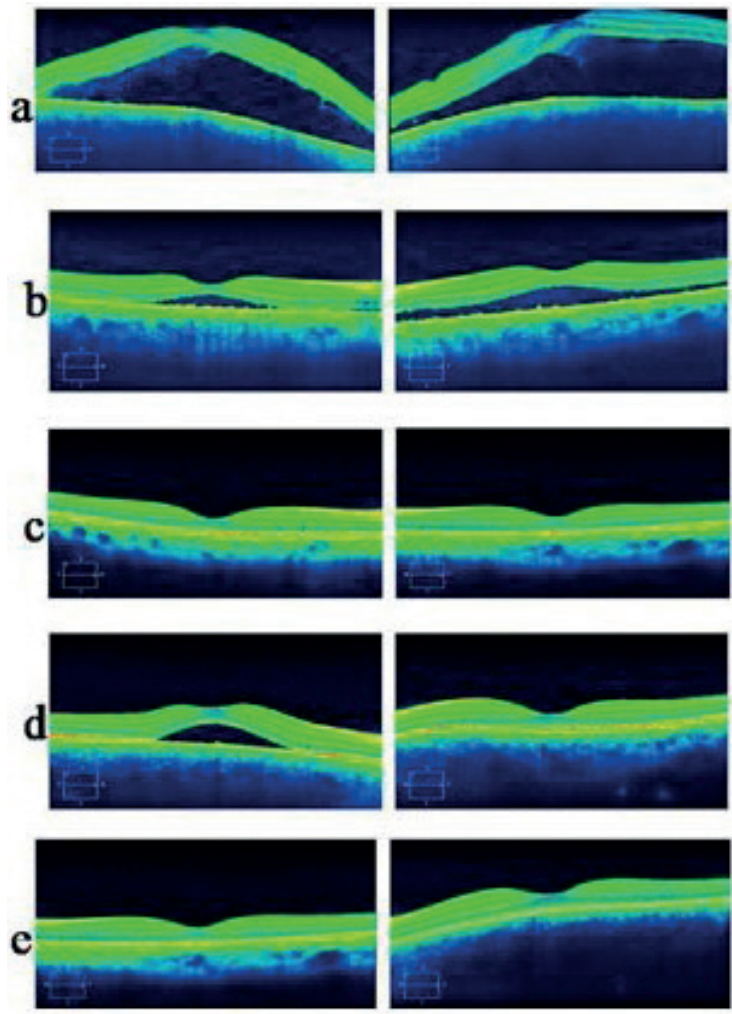

Fig. 3. a-OCT images at the first visit: Serous retinal detachment in both eyes. b-OCT images at the seventh day of steroid treatment: OCT images showed decreased subretinal fluid in both eyes. c-OCT images at first month follow-up visit: Subretinal fluid was lost in OCT images of both eyes. d-OCT images at second month follow-up visit: Subretinal fluid was seen again in OCT image of the right eye. e-OCT images at follow-up visit two weeks after low dose azathioprine treatment: Subretinal fluid was lost in the right eye.

disappearance of the subretinal fluid in both of the eyes (Fig. 3c). However, the patient had complaints and findings of weight gain and the onset of Cushingoid-like fat tissue distribution, thus, she was consulted to the department of endocrinology. The endocrinology department planned a gradual reduction and termination of the oral steroid treatment. The steroid treatment was gradually decreased. While she was at the dose of $8 \mathrm{mg} /$ day at the follow-up visit in the second month, the patient's visual complaints emerged again, and her BCVA was measured as 20/25 in the right, and 20/20 in the left eye. SD-OCT image of the right eye 


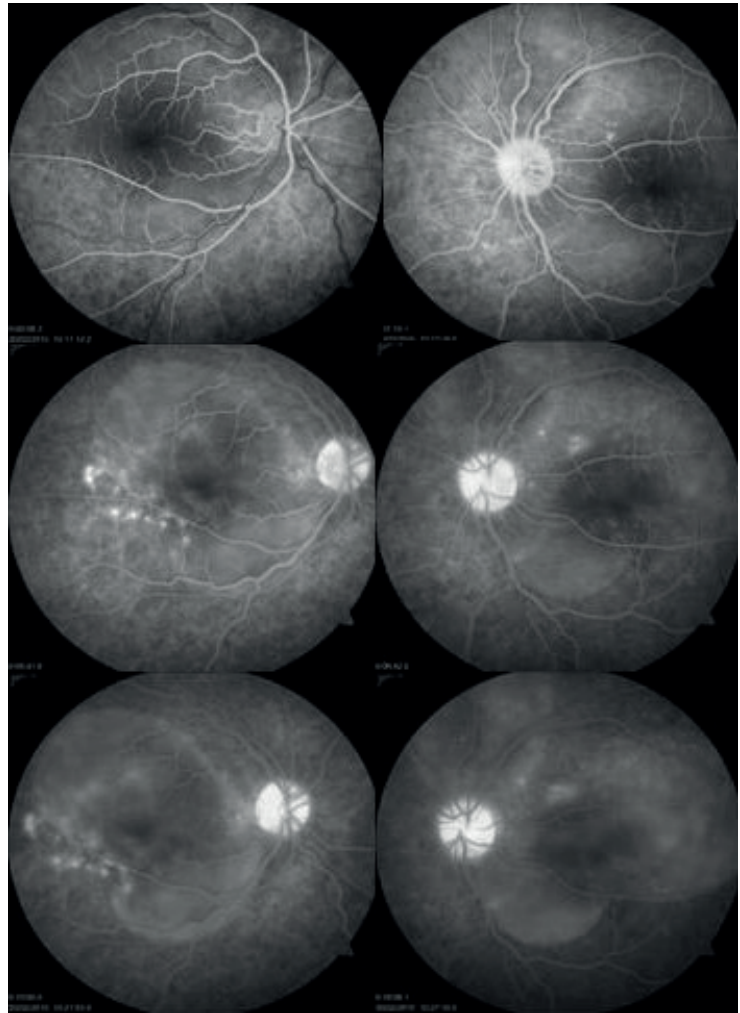

Fig. 4. Fundus fluorescein angiography (FFA) images at the first visit: FFA demonstrated serous retinal detachment of posterior pole and papillitis in both eyes.

showed subretinal fluid again (Fig. 3d). The patient was re-consulted to the department of pediatrics, and after monitoring her hemogram and biochemistry results, the current treatment was continued with a low-dose steroid (prednisolone $4 \mathrm{mg} /$ day) and $50 \mathrm{mg}(1 \mathrm{mg} / \mathrm{kg} /$ day) azathioprine. At the follow-up visit, which was two weeks after the last visit, bilateral BCVA was $20 / 20$, and the subretinal fluid disappeared again on the SD-OCT image of the right eye (Fig. 3e). Subfoveal choroidal thickness of both eyes was measured again and there was thinning in the choroidal thickness of both eyes according to the first visit $(315 \mu, 311 \mu$ in the right eye and the left eye, respectively). Her oral steroid dose was completely stopped and she had no complaints and residual findings of the side effects of steroids. The patient was treated with only low-dose azathioprine $(50 \mathrm{mg} /$ day) during the rest of the first year and no recurrence was observed in the last 3 years.
An informed consent form was obtained from the patient and her family for the publication of this case.

\section{Discussion}

The etiology of VKHS is still unknown, although an immune response against melanocytes has been held responsible for the pathogenesis of the disease. The immune response to melanocytes in the uveal system results in bilateral panuveitis, optic disc hyperemia and serous retinal detachment. ${ }^{14}$ There is no specific diagnostic method for VKH disease. The diagnosis of the disease is made after the exclusion of other causes of uveitis. Therefore, VKHS is diagnosed using established diagnostic criteria, based on clinical findings. ${ }^{2}$

The differential diagnosis includes infectious agents such as syphilis, tuberculosis, toxoplasma, AIDS; systemic inflammatory diseases such as Behçet's disease, systemic lupus erythematosus, rheumatoid arthritis, Wegener's granulomatosis, ulcerative colitis and sarcoidosis; ocular diseases such as sympathetic ophthalmia, intraocular lymphoma, central serous chorioretinopathy, posterior scleritis, uveal effusion syndrome, malignant hypertensive retinopathy. ${ }^{15}$ Additionally, starry sky appearance is one of the characteristic features of VKHS, however starry sky appearance on fluorescein angiography can be seen in numerous conditions, including sympathetic ophthalmia, central serous chorioretinopathy, posterior scleritis, leukemia, DIC, malignant hypertension. Juvenile rheumatoid arthritis is the most common etiology of pediatric anterior uveitis and disease associated with ANA. ${ }^{16}$ Clinical presentation of VKH may mimic sympathetic ophthalmia but there is no history of ocular trauma or ocular surgery in patients with VKHS. ${ }^{17}$ Serum angiotensin converting enzyme level, a chest radiograph can aid in the differential diagnosis for sarcoidosis. In addition, retinal vascular findings in Behçet's disease and sarcoidosis are more evident. ${ }^{15}$ The RPE sub-infiltrates 
seen in B cell lymphoma are much larger and fewer than the yellow infiltrates seen in VKHS. ${ }^{15}$ Ultrasonography can help differentiate VKHS from posterior scleritis. ${ }^{18}$ Patients with idiopathic uveal effusion syndrome do not have intraocular inflammation; and exudative retinal detachments tend to be chronic and they are resistant to medical therapy such as corticosteroids or antimetabolites. ${ }^{19}$

Our patient had no history of penetrating ocular trauma or surgery before the onset of the first uveitis. In our patient's laboratory tests and imaging methods, no findings or test results were found for diseases above stated for differential diagnosis. In our case, early phase findings of the disease were bilaterally present and also had neurological findings. Therefore, according to the revised diagnostic criteria of VKHS; our patient was defined as incomplete VKHS. $^{20}$ If our patient had symptoms of alopecia, poliosis or vitiligo, we could describe the disease as complete, but these findings were not observed in the last 3 years. We can associate the absence of these symptoms with early diagnosis and correct treatment.

Multiple treatment methods have been tried for VKHS, including corticosteroid, cyclosporine, antimetabolites, and alkylating agents. ${ }^{21}$ Insufficient immunosuppressive therapy will cause relapses in the disease and the disease will tend to become chronic. Early and high-dose systemic corticosteroids are used to suppress inflammation in the acute stage of the disease. High-dose corticosteroids are associated with side effects such as hyperglycemia, hypertension, immunodeficiency, weight gain, cushing syndrome and secondary glaucoma. ${ }^{22}$ In the literature; due to side effects or in resistant cases, agents such as cyclosporine, azathioprine, adalimumab, methotrexate, mycophenolate have been added to the treatment (Table I).

Azathioprine, is usually used in the treatment of immunological diseases such as inflammatory bowel disease (IBD) and autoimmune hepatitis in children. ${ }^{23}$ In the literature; it has been reported that azathioprine is combined with steroids in the treatment of children with VKHS. ${ }^{24}$ We used low dose steroids $(4 \mathrm{mg}$ / day) and systemic azathioprine (50 mg/day) to overcome side effects and relapses. Two weeks after the indicated treatment, steroid treatment was discontinued. Azathioprine has been shown to be effective by some authors in patients with corticosteroid intolerance. ${ }^{25}$ Azathioprine can cause some serious side effects, such as myelosuppression and gastrointestinal disorders. However, these side effects are unusual with low dose use $(1 \mathrm{mg}$ / $\mathrm{kg} /$ day) compared to high doses. ${ }^{22}$ During the first 3 years of follow-up, we did not observe any recurrence, sunset glow fundus appearance and dermatological problems. These findings are consistent with previous literature, which concluded that VHS patients who were diagnosed and treated immediately did not develop dermatologic lesions. ${ }^{26}$

Finally, VKHS is a disease rarely presented in young patients, and it can be misdiagnosed due to its rarity. Because of the major complications of the disease that threaten the ocular vision, patients should be treated as soon as possible with corticosteroids and/or immunosuppressive drugs. In conclusion, early diagnosis and selection of timely and appropriate treatment protocol provided a good visual prognosis without serious complications in the patient. Further case series and randomized-controlled studies with large sample sizes are needed to enlighten the other probable effective therapies or their side effects also in the young VKHSs.

\section{Declaration of patient consent}

The authors certify that they have obtained all appropriate patient consent forms. In the form the patient(s) has/have given his/her/ their consent for his/her/their images and other clinical information to be reported in the journal. The patients understand that their names and initials will not be published and due efforts will be made to conceal their identity, but anonymity cannot be guaranteed. 


\section{Author contribution}

Study conception and design: Gamze Yıldırım, Cemal Çavdarlı; data collection: Mehmet Numan Alp; analysis and interpretation of results: Emine Yıldız Özdemir; draft manuscript preparation: Gamze Yıldırım.

All authors reviewed the results and approved the final version of the manuscript.

\section{Conflicts of interest}

There are no conflicts of interest.

\section{REFERENCES}

1. Yoshida A, Tominaga S, Kawashima H. Juvenile Vogt-Koyanagi-Harada disease in which good visual prognosis was derived from swift definitive diagnosis. Case Rep Ophthalmol Med 2016; 2016: 7936729 .

2. Abu El-Asrar AM, Al-Kharashi AS, Aldibhi H, AlFraykh H, Kangave D. Vogt-Koyanagi-Harada disease in children. Eye (Lond) 2008; 22: 1124-1131.

3. Tabbara KF, Chavis PS, Freeman WR. VogtKoyanagi-Harada syndrome in children compared to adults. Acta Ophthalmol Scand 1998; 76: 723-726.

4. AlQahtani DS, Al Shamrani M. Case report of a 4-year-old child with complicated Vogt-KoyanagiHarada at a tertiary eye hospital. Middle East Afr J Ophthalmol 2019; 26: 117-119.

5. Katsuyama A, Kusuhara S, Awano H, Nagase H, Matsumiya W, Nakamura M. A case of probable Vogt-Koyanagi-Harada disease in a 3-year-old girl. BMC Ophthalmol 2019; 19: 179.

6. Khan F, Zahid S, Raza SS, Iqbal M. A case of Vogt Koyanagi Harada disease in a 16 year old girl. J Pak Med Assoc 2017; 67: 1759-1761.

7. Takada S, Tanaka R, Kurita N, Ishii K, Kaburaki T. Vogt-Koyanagi-Harada disease in 3-year-old boy. Clin Exp Ophthalmol 2015; 43: 593-594.

8. Bilgiç Ö, Gündüz A, Kaya K. Vogt-Koyanagi-Harada disease in an adolescent boy. Pediatr Dermatol 2014; 31: 99-101.

9. Vergaro R, Cordelli DM, Miniaci A, et al. Cerebral ischemic involvement in Vogt-Koyanagi-Harada disease. Pediatr Neurol 2014; 51: 119-122.

10. Bušányová B, Tomčíková D, Gerinec A. VogtKoyanagi-Harada syndrome in children - a case report. Cesk Slov Oftalmol 2013; 69: 81-86.
11. Hernandez C, LePoole C, Tessler HH. VogtKoyanagi-Harada syndrome in a 6-year-old Hispanic boy. Pediatr Dermatol 2012; 29: 191-194.

12. Venaille A, Palombi K, Templier I, et al. VogtKoyanagi-Harada syndrome: a serious and rare emergency in children. Arch Pediatr 2011; 18: 33-36.

13. Benfdil N, Baha Ali T, Jellab B, Moutaouakil A. Vogt Koyanagi Harada syndrome in children: diagnosis and management. Bull Soc Belge Ophtalmol 2010; 15-18.

14. Martin TD, Rathinam SR, Cunningham ET Jr. Prevalence, clinical characteristics, and causes of vision loss in children with Vogt-Koyanagi-Harada disease in South India. Retina 2010; 30: 1113-1121.

15. Damico FM, Kiss S, Young LH. Vogt-KoyanagiHarada disease. Semin Ophthalmol 2005; 20: 183190.

16. Pivetti-Pezzi P. Uveitis in children. Eur J Ophthalmol 1996; 6: 293-298.

17. Cunningham ET Jr, Kilmartin D, Agarwal M, Zierhut M. Sympathetic ophthalmia. Ocul Immunol Inflamm 2017; 25: 149-151.

18. Kumar A, Ghose A, Biswas J, Majumder PD. Clinical profile of patients with posterior scleritis: a report from Eastern India. Indian J Ophthalmol 2018; 66: 1109-1112.

19. Valmaggia C, Helbig H, Fretz C. Uveal effusion syndrome. Klin Monbl Augenheilkd 2007; 224: 317319.

20. Read RW, Holland GN, Rao NA, et al. Revised diagnostic criteria for Vogt-Koyanagi-Harada disease: report of an international committee on nomenclature. Am J Ophthalmol 2001; 131: 647-652.

21. O'Keefe GA, Rao NA. Vogt-Koyanagi-Harada disease. Surv Ophthalmol 2017; 62: 1-25.

22. Lodhi SA, Reddy JL, Peram V. Clinical spectrum and management options in Vogt-Koyanagi-Harada disease. Clin Ophthalmol 2017; 11: 1399-1406.

23. Citterio-Quentin A, Moulsma M, Gustin MP, Lachaux A, Boulieu R. ITPA activity in children treated by azathioprine: relationship to the occurrence of adverse drug reactions and inflammatory response. Basic Clin Pharmacol Toxicol 2018; 122: 588-595.

24. Berker N, Ozdamar Y, Soykan E, Ozdal P, Ozkan SS. Vogt-Koyanagi-Harada syndrome in children: report of a case and review of the literature. Ocul Immunol Inflamm 2007; 15: 351-357.

25. Kim SJ, Yu HG. The use of low-dose azathioprine in patients with Vogt-Koyanagi-Harada disease. Ocul Immunol Inflamm 2007; 15: 381-387.

26. Cunningham ET Jr, Rathinam SR, Tugal-Tutkun I, Muccioli C, Zierhut M. Vogt-Koyanagi-Harada disease. Ocul Immunol Inflamm 2014; 22: 249-252. 